\title{
Variation in the Gut Microbiota of Common Marmosets: Differences with Colony of Origin and Integration
}

6 Rachel E. Cooper, ${ }^{\mathrm{a}}$ Lisa M. Mangus, ${ }^{\mathrm{a}}$ Jessica Lynch, ${ }^{\mathrm{b}}$ Kayla Schonvisky, ${ }^{\mathrm{b}}$ Justin R. Wright, ${ }^{\mathrm{c}}$,

7 Christopher J. McLimans ${ }^{\text {c }}$, Hoi Tong Wong ${ }^{c}$, Jeremy Chen See ${ }^{c}$, Regina Lamendella, ${ }^{c}$ Joseph

10 aDepartment of Molecular and Comparative Pathobiology, Johns Hopkins University, Baltimore,

11 MD, USA

$12{ }^{b}$ Department of Biomedical Engineering, Johns Hopkins University, Baltimore, MD, USA

$13{ }^{\mathrm{c} W r i g h t L a b s ~ L L C ., ~ H u n t i n g d o n, ~ P A, ~ U S A ~}$

14

15 \#Corresponding author: jmankows@jhmi.edu

16 Word count (abstract): 249

17 Word count (text): 4321 
Abstract [248/250 words]

Characterization of the gut microbiome may aid understanding and management of

natural and experimental disease states in research animals, thereby promoting reproducibility. In

21 this study, the rectal bacterial communities of three separate common marmoset (Callithrix

22 jacchus) breeding colonies were defined using 16S rRNA sequencing of rectal swab samples.

23 Study animals originated from two German colonies and a United States colony (JHU). The two

24 German cohorts, previously fed the same diet, were imported into the JHU facility; they were

25 then isolated, transitioned onto JHU diet, and then moved into rooms housing JHU animals. To

26 dissect the contributions of diet and integration in shaping the rectal bacterial community,

27 samples were collected from German origin marmosets upon JHU arrival (baseline), following

28 diet transition (100 d), and following cohousing (390 d). Baseline and $390 \mathrm{~d}$ samples were

29 collected from stably maintained JHU marmosets. Bacterial community composition was distinct

30 between all three cohorts at baseline, suggesting that factors other than primary diet confer

31 significant differences between captive populations. Beta-diversity of the animals from the two

32 German colonies converged by $100 \mathrm{~d}$ but remained distinct from JHU sample beta-diversity

33 throughout the 390-d study, indicating that diet had greater influence on bacterial community

34 composition than did housing animals within the same room. Our results demonstrate substantial

35 differences in gut bacteria between different captive marmoset colonies, with persistence of these

36 differences following husbandry standardization and housing integration. Goals of rigor and

37 reproducibility in research underscore the need to consider microbial differences between

38 marmosets of diverse origin. 


\section{Importance [141/150 words]}

43 research reproducibility in animal studies. As use of common marmosets as animal models of

44 human diseases expands, evaluating the marmoset gut bacterial community will be critical for

45 interpreting research findings, especially as marmosets are prone to gastrointestinal

46 inflammation. In this study, using 16S rRNA sequencing of rectal swab samples, we compared

47 bacterial community among three captive colonies of marmosets at baseline and following

48 importation of cohorts from two of the colonies into the third colony. Diet history had sustained

49 influence on bacterial community composition, while housing the animals within the same room

50 over a period of eight months did not appear to be a major factor. These persistent differences in

51 marmoset gut bacterial community highlight the need for careful consideration of animal origin

52 as a variable in marmoset research studies. 


\section{Introduction}

Interactions between commensal microbes and their human and animal hosts have widereaching effects on physiology. In the context of animal research, these effects must be

57 considered in the development and maintenance of robust model systems. In particular, the

58 microbiome of the gut comprises the largest and most diverse microbial population in mammals,

59 and characterization of this system will be helpful in understanding and managing natural and

60 experimental disease states in research animals. The common marmoset (Callithrix jacchus) is

61 an increasingly utilized translational model within the fields of behavior, neuroscience, nutrition,

62 and aging. However, it is unknown whether and to what extent the gut microbiota differs

63 between marmosets of differing captive origin. Importantly, limited availability of marmosets

64 for biomedical research often requires animals to be obtained from a variety of colonies for

65 inclusion in a single study cohort. The need to maintain genetically diverse breeding pools also

66 prompts transfer of marmosets between colonies, with unknown implications for ongoing

67 research models. ${ }^{1}$

Broadly, environmental factors are a key source of microbial variability among

69 individuals. In one study of the gut bacterial microbiota in multiple marmoset species and hybrid

70 types, the degree of captivity - captive-born vs. wild-born captive vs. wild - had a stronger

71 overall effect on the microbial composition than did host genetics. ${ }^{2}$ Importantly, there is reason

72 to believe that even conditions of captivity that are relatively similar between colonies can yield

73 significantly differential gut microbial community composition. In inbred mice populations

74 originating from different vendors, or arriving at different facilities with standardized husbandry

75 practices, significant gut microbial variation exists and has been shown to impact research

76 outcomes and disease phenotype. ${ }^{3-7}$ Captive care and husbandry for marmosets has been an 
77 ongoing challenge in maintaining these arboreal, exudativorous new world primates in captive

78 settings, and practices to date are unstandardized in comparison to those for mice used in

79 research. ${ }^{8}$ Given variation reported in mouse models in stable and changing conditions of

80 captivity, it is probable that (1) the gut microbiota of captive common marmosets varies widely

81 between colonies, and (2) there is a significant degree of gut microbial community plasticity

82 following minor (i.e. captive to captive) environmental change in the common marmoset.

Recent attention to the variation in husbandry practices has underscored the relevance of

84 such variation in research reproducibility. ${ }^{8,9}$ Strikingly, one research group found a $25 \%$

85 reduction in clinically evident recombinant protein-induced experimental autoimmune

86 encephalomyelitis (EAE) in common marmosets fed a yoghurt-based supplemental diet that

87 differed from the standard colony enrichment; the primary diet for both groups was the same.

88 Divergence in the fecal bacterial microbiota (bacteriota) of the two groups was minimal at seven

89 weeks following implementation of varied dietary supplementation, but lower abundance of

90 Bifidobacterium spp. and higher abundance of Collinsella tanakaei was noted in the traditional

91 supplementation (non-yoghurt-based) group three weeks following EAE induction, suggesting

92 interplay between diet, the microbiota, and immune function in this model. ${ }^{9}$ This is not an

93 isolated example. In humans and other species, the gut microbiome has been widely implicated

94 in development and modulation of the nervous system via the gut-brain axis, ${ }^{10-16}\left\{\mathrm{O}^{\prime}\right.$ Mahony,

95 2015 \#1505;Zheng, 2019 \#1483;Zhu, 2019\#1474;Hsiao, 2013 \#1726\} and in progression of

96 both neurodevelopmental and neurodegenerative disease phenotypes. ${ }^{9,17-19}$ Particularly with the

97 emergence of transgenic, induced, and spontaneous common marmoset models of human

98 neurodegenerative diseases including polyglutamine diseases (e.g. Huntington's disease and

99 spinocerebellar ataxia), Alzheimer's disease, Parkinson's disease, schizophrenia, and 
100 amyotrophic lateral sclerosis, ${ }^{20,21}$ it is imperative that the research community consider gut-

101 microbiome-brain interactions in the context of these translational models.

104 segments of the gut in nonhuman primates. ${ }^{22}$ We examined whether and to what extent (1) the

105 gut bacterial microbiota of marmosets originating from separate captive colonies displayed

106 significant deviation; (2) institutional transfer conferred significant differences in the gut fecal

107 microbiota of these animals; and (3) the gut bacterial microbiota in adult marmosets within a

108 representative, stable captive research colony maintained stability over time. For 390 d following

109 importation of healthy cohorts from an established academic research German colony (HHG)

110 and an established primate research center Germany colony (DPG), we assessed and compared

111 rectal bacterial microbiota of these marmosets and of healthy colony animals from an established

112 United States academic research colony (JHU) of over 120 individuals.

113 Based on previous work indicating environmental and genetic effects on the gut

114 microbiota in mammalian species, ${ }^{23,24}$ we hypothesized that the gut bacterial communities of the

115 three captive common marmoset populations, as assessed by $16 \mathrm{~S}$ rRNA sequencing and

116 bioinformatics analysis, would differ. We hypothesized that greater discrepancy would exist

117 between the German-origin (DPG, HHG) and JHU groups than between DPG and HHG at

118 baseline, given (1) the same primary diet fed to DPG and HHG, with a different primary diet fed

119 to JHU, and (2) more recent microbial and genetic transmission between DPG and HHG

120 populations. In evaluating longitudinal changes within each of these three populations, we

121 hypothesized greater change in bacterial community structure of the German-origin groups over

122 time as compared to the JHU group, which underwent no husbandry or diet change. To 
123 determine the relative contributions of variables relevant to captive research colonies,

124 correlational and diversity analyses were conducted considering the following host

125 characteristics: sex, age, weight, and housing status (single versus pair- or group-housed). 


\section{Results}

127 Sequencing Summary. In total, over 6.5 million 16S rRNA gene sequences were obtained across

128 the entire dataset post-quality filtration and denoising. A range of 9,600-129,000 filtered

129 sequences per sample were obtained, with a minimum requirement of each sample yielding at

130 least 5000 sequences to be included in downstream diversity analyses. Over 1300 unique

131 amplicon sequence variants (ASVs) were identified across all processed samples.

132 Evaluation of Rectal Bacterial Community Composition at Baseline.

Sample Diversity (alpha diversity) by Facility Origin. There was no difference in gut

134 bacterial species richness between the three cohorts at baseline (observed ASVs, P > 0.05; Figure

135 2A). There was increased bacterial evenness in the JHU cohort compared to the HHG cohort

136 (Heip's evenness, $\mathrm{p}=0.006$; Figure 2B). Bacterial evenness in the DPG cohort was intermediate

137 and did not differ from that of HHG or JHU animals (Heip's evenness, $\mathrm{p}>0.05$; Figure 2B).

138 Sample Composition (beta diversity) by Facility Origin. Facility origin was associated with

139 significant differences in rectal bacterial community composition (Analysis of similarities:

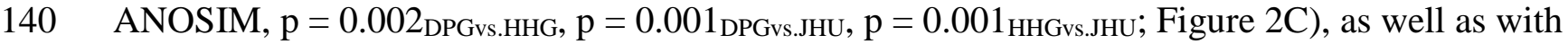

141 observed differences in relative abundance of individual taxa (Figure 2D; Table 1). The majority

142 of DPG samples were dominated by Bacteroidetes (66.7\%) or Campylobacterota (27.8\%), most

143 HHG samples were dominated by phyla Proteobacteria (53.3\%) or Bacteroidetes (40\%), and

144 most JHU samples were dominated by Bacteroidetes (77.8\%) or Fusobacteria (11.1\%) (Table 1).

145 Campylobacterota (or Epsilonbacteraeota) is a recently-proposed phylum comprising members

146 of the class Epsilonproteobacteria within the phylum Proteobacteria; genera include

147 Campylobacter and Helicobacter. ${ }^{25,26}$ 


\section{HHG (100 d), and Integration of All Populations (390 d).}

Sample Diversity over Time. In the DPG cohort, there was higher bacterial richness noted at $390 \mathrm{~d}$ compared to $100 \mathrm{~d}(\mathrm{p}=0.01)$; however, evenness in the DPG cohort did not

152 significantly differ between any time points (Figure 3A-B). There was no change in richness or 153 evenness between baseline and the $390 \mathrm{~d}$ time point in HHG marmosets (observed ASVs, $\mathrm{p}>$

$154 \quad 0.05$; Heip's evenness, $\mathrm{p}>0.05$; Figure 3A-B). However, in this cohort there was an increase in 155 bacterial richness between the $100 \mathrm{~d}$ and $390 \mathrm{~d}$ time points (Observed ASVs, $\mathrm{p}=0.003$ ) and an 156 increase in evenness between the baseline and $100 \mathrm{~d}$ time points (Heip's evenness, $\mathrm{p}=0.045$ ). In 157 general, trends suggest a nadir in richness and a peak in evenness at the $100 \mathrm{~d}$ time point for the 158 HHG cohort.

Following integration with the arrived German cohorts (390 d), the bacterial population of JHU animals decreased in evenness (Heip's evenness, $p=0.002$; Figure 4B). There was a 161 non-significant trend suggesting an increase in richness (Observed ASVs, $p>0.05$; Figure 4A).

165 composition differed from baseline to $390 \mathrm{~d}$ (ANOSIM, $\mathrm{p}=0.011 \mathrm{JHU}, \mathrm{p}=0.002_{\mathrm{HHG}}, \mathrm{p}=$

166 0.02 ${ }_{\mathrm{DPG}}$; Figures 3C-D, 4C-D; Table S2). In pairwise comparisons of each of the three time

167 points for DPG and HHG cohorts, significant differences for each population group were

168 observed between every time point, except for between $100 \mathrm{~d}$ and $390 \mathrm{~d}$ in the DPG cohort

169 (ANOSIM; Table S2). 
At $100 \mathrm{~d}$ and $390 \mathrm{~d}$, there was no significant difference in bacterial community

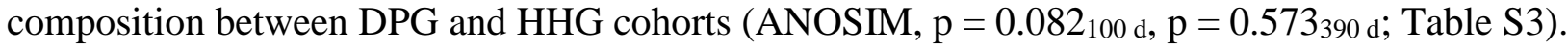

172 At 390 d, the bacterial microbiota of DPG and HHG marmosets remained distinct from that of

173 JHU marmosets (ANOSIM; $\mathrm{p}=0.006_{\text {DPGvs.JHU, }} \mathrm{p}=0.004_{\text {HHGvs.JHU; }}$ Table S3).

At 390 d, there were persistent differences at the phylum, class, order, and family levels; there were 39 differential taxa, compared to 25 differential taxa at baseline (Linear discriminant analysis: LDA > 2.5; Figures 2D, 5D). At 390 d, most samples from all cohorts were

177 predominated by the phylum Bacteroidetes (DPG: 563\%, HHG: 50\%, JHU: 76.9\%); phyla of 178 secondary predominance included Epsilonbacteraeota (37.5\%) for DPG marmosets, Firmicutes 179 (21.4\%) and Fusobacteria (21.4\%) for HHG marmosets, and Fusobacteria (23.1\%) for JHU marmosets (Table 1).

Evaluation of Study Subject Characteristics.

Sample Composition by Sex. At 390 d, with all marmoset cohorts grouped for analysis,

183 there was no effect of sex (male; $n=20$ vs. Female; $n=23$ ) on overall bacterial community 184 composition (ANOSIM, $q=0.623, \mathrm{p}=0.623$; Figure $6 \mathrm{C}$ ). However, seven bacterial taxa were 185 significantly enriched in male marmosets (LDA > 2.5, Figure 6D).

187 for analysis, there was no effect of housing status (singly vs. pair- or group-housed) on bacterial 188 community composition (ANOSIM, $\mathrm{p}=0.38$ ).

Sample Composition by Age. At 390 d, with all marmoset cohorts grouped for analysis,

190 two bacterial taxa varied significantly with age; both increased in abundance as animal age

191 increased (Spearman correlation: genus Phascolarctobacterium, $r=0.380, \mathrm{p}=0.017$; family

192 Peptostreptococcaceae $r=0.372, \mathrm{p}=0.019$; Table S4). 
Sample Composition by Body Mass. At 390 d, with all marmoset cohorts grouped for

194 analysis, 13 bacterial taxa varied significantly with body mass. Six taxa (Clostridium sensu

195 stricto, Fusobacterium mortiferum, Butyricicoccus, Cellulosilyticum, Enterococcus, and Phocea

196 massiliensis) correlated positively (Spearman: $r=0.322-0.410, \mathrm{p}<0.05$; Table S5); 7 taxa

197 (Bifidobacteriaceae, Bifidobacterium aesculapii, Lachnoclostridium, Muribaculaceae,

198 Atopobiaceae, Peptostreptococcaceae, and Phascolarctobacterium) correlated negatively

199 (Spearman: $r=-0.328--0.404, \mathrm{p}<0.05$; Table S5). There were no significant correlations above

200 the order taxonomic level. 


\section{Discussion}

The results of this study emphasize the importance of animal source as a potential

204 variable for research. We found significant baseline differences in the rectal bacterial community composition of two different captive populations (DPG, HHG) originating from the same country, fed the same primary diet, and with limited cross-institutional animal transfer in recent years. Although there were no differences in overall bacterial community structure (betadiversity) by $100 \mathrm{~d}$ following colony integration, significant differences in the presence of certain bacterial taxa (e.g. Helicobacter jaachi) persisted for the length of the study. Rectal bacterial differences were even more persistent between populations that had previously been fed different

211 primary diets and had different captive country origin (DPG, HHG vs JHU); bacterial

212 community composition between such populations remained significantly different for over one

213 year. Greater likelihood of genetic similarity between DPG and HHG populations is unlikely to

214 explain these results; previous studies in nonhuman primates have found that environment - diet

215 in particular - appears to be much more important than host genetics in predicting the

216 composition of the gut microbiota. ${ }^{2,24}$

390 d after importation, bacterial community composition remained distinct between the

218 imported population groups (DPG, HHG) and the stable population group (JHU). These results

219 suggest a level of long-term stability of the gut microbiota in captive marmosets when moved

220 between institutions and subjected to diet change. Over the course of the study - while all

221 animals were housed at JHU - the rectal bacterial microbiota of the two German cohorts

222 converged, predominantly between the baseline and $100 \mathrm{~d}$ time points. This suggests that diet

223 change and standardization of other environmental parameters (e.g. water source, non-edible

224 enrichment items, air quality) were instrumental in altering the microbiota. Interestingly, though 
225

226

227

228

sample composition was not significantly different between the DPG and HHG groups by $100 \mathrm{~d}$, each population retained distinctive microbial signatures throughout the study. DPG marmoset samples, for example, remained uniquely enriched in Epsilonproteobacteria (proposed phylum Campylobacterota), ${ }^{25,26}$ specifically Helicobacter jacchi, ${ }^{27}$ through $390 \mathrm{~d}$.

Although differences between the German cohorts and the JHU cohort persisted through $390 \mathrm{~d}$, we did not find evidence to attribute this fully to long-term stability in the JHU gut bacterial microbiota. Rather, significant alterations in bacterial community structure developed in the JHU group over time. The magnitude of alteration was surprising, given that animals in the JHU cohort did not undergo any substantial changes in husbandry or care practices other than introduction of two relatively small cohorts, comprising 57 marmosets total, into three adjacent housing rooms containing an existing colony of approximately 120 marmosets. One likely explanation for this alteration is that the introduction of new marmosets induced changes in microbial community structure, either through introduction of novel bacteria or other community-altering flora (e.g. phages, fungi) or via other physiological effects (e.g. stress). Other possibilities include random change over time (i.e. drift), or aging of animals within the cohort.

We conducted correlational analysis in order to evaluate the possibility of age as a confounder in this longitudinal study. In humans, aging has been associated with changes in the gut microbiome; with increased age, there has been demonstrated a lack of diversity in the core bacterial taxa and a loss of microbiota stability. ${ }^{28-30}$ Among adult marmosets, we found minimal impact of age on composition of the gut bacterial population, with two taxa within the phylum Firmicutes, genus Phascolarctobacterium and family Peptostreptococcaceae, demonstrating a weak positive correlation with age. These findings differ from those previously reported in 
marmosets; one recent study of 20 captive common marmosets found increased relative

249 abundance of Proteobacteria and decreased Firmicutes in geriatric marmosets (> 8 years of age)

250 compared to young adults. ${ }^{31}$ Findings in the current study, which demonstrated only two taxa

251 that correlated in abundance with advancing age, may be attributable to the relatively limited

252 diversity of ages represented by our study subjects (2.1 years -7.82$)$; this range excludes

253 geriatric animals, and represents the large majority of marmosets actively used in research.

In this study population of clinically healthy marmosets, body mass was correlated with

255 abundance of a number of bacterial phyla in the gut microbiota. Within Firmicutes, Clostridium

sensu stricto, Butyricicoccus, Cellulosilyticum, Enterococcus, and Phocea massiliensis were

257 present in increased abundance in common marmosets with higher body mass while

258 Lachnoclostridium, Peptostreptococcaceae, and Phascolarctobacterium were present in

259 decreased abundance. Within the phylum Bacteroidetes, the family Muribaculaceae was found to

260 weakly negatively correlate with body mass. In humans, an increased Firmicutes:Bacteroidetes

261 ratio has been associated with increased obesity risk; ${ }^{32}$ at the phylum level, we did not identify

262 this in our marmoset population. Abundance of Bifidobacteriaceae (phylum: Actinobacteria),

263 including the species Bifidobacterium aesculapii, was negatively correlated with body mass in

264 the present study. Members of this taxonomic family have been associated with anti-obesity

265 effects in mice and humans, ${ }^{33,34}$ and are found in decreased abundance in obese humans. ${ }^{35,36}$

266 These preliminary findings in healthy marmosets, taken together with supporting evidence in

267 other species, suggests utility in further investigation into the relative abundance and role of

268 Bifidobacteriaceae in the gut of obese marmosets.

269 We detected no influence of sex on overall bacterial community composition;

270 nonetheless, several individual taxa, including families Cardiobacteriaceae (phylum: 
271 Proteobacteria), Pasteurellaceae (phylum: Proteobacteria), and Enterococcaceae (phylum:

272 Firmicutes), were relatively enriched in male marmosets. Studies in mice and humans have

273 demonstrated the presence of sex differences, though such differences appear relatively minor; in

274 studies of inbred mice, for example, strain identity has accounted for greater variation than has

275 sex. ${ }^{37-39}$

276 The most common and growing use of common marmosets in research is for neuroscience

277 and behavior studies, including development of transgenic animals to model neurodevelopmental

278 and neurodegenerative disease. Scientific literature has broadly established the importance and

279 relevance of the microbiome-brain-gut axis $;^{12-19}$ therefore, particularly given the protracted

280 microbiota differences noted in this study, investigators should be aware of the potentially

281 confounding effects of the gut microbiome in the use of differently originating captive common

282 marmosets in development of research models. Transport of animals from one institution to

283 another, even if marmosets remain on the same diet, may have effects on the microbiome and

284 therefore the research model. Likewise, movement of new animals into an existing colony should

285 be performed with caution and understanding that the gut microbiome of existing colony animals

286 may experience significant alteration. Due to the increasing demand for marmosets in research

287 and limited availability of animals from any single colony, these results will be relevant to most

288 captive research colonies within the United States and elsewhere. 


\section{Materials and Methods}

291 Study population: Adult animals belonging to three captive populations of common marmosets

292 were examined in the present study. At the time of sample collection, all animals were housed at

293 Johns Hopkins University, an AAALAC-accredited institution; all procedures were approved by

294 the Johns Hopkins University IACUC. Animals were allowed ad libitum access to chlorine

295 dioxide-treated water (Quip Labs) delivered in water bottles. Marmosets were fed one of two

296 complete commercial callitrichid diets, detailed below, in addition to daily supplementation with

297 some combination of seeds, dried fruit, wax worms, cheerios, tofu, chickpeas, and fresh fruit

298 (e.g. banana, pineapple, kiwi).

JHU Population: Approximately 120 animals ( $0-12.4$ years old) were housed in family groups, in pairs, or singly as part of a long-standing breeding and experimental colony at Johns

Hopkins University. Animals actively engaged in experimental use participated in awake

behavioral experiments with or without cranial implants, and/or anesthetized neurophysiologic

experiments with cranial implants. This population was fed a primary diet of Advanced

304 Protocol® Callitrichid Diet 5LK6 (Purina LabDiet@, St. Louis, MO). This diet contained at least

$30521 \%$ crude protein, at least $7 \%$ crude fat, up to $2.5 \%$ crude fiber, $12 \%$ starch, $35 \%$

306 monosaccharides/disaccharides, $1.1 \%$ calcium, $0.64 \%$ phosphorus, and 6,600 IU/kg Vitamin $\mathrm{D}_{3}$.

HHG population: Seventeen animals $(1.7-6.8$ years old) originating from the University

308 of Düsseldorf (Heinrich-Heine-Universität Düsseldorf, Düsseldorf, Germany) were housed

309 singly or in groups for the duration of import and quarantine. At the original institution, during

310 CDC import quarantine (31 d), and during quarantine at Johns Hopkins University, this

311 population was fed a primary diet of Mar V3843 (ssniff®, Soest, Germany). This diet contained 
$31226 \%$ crude protein, $7 \%$ fat, $2.5 \%$ crude fiber, $1 \%$ calcium, $0.7 \%$ phosphorus, and 3,000 IU/kg

313 vitamin $\mathrm{D}_{3}$

314 DPG population: Forty animals $(2.8-8.7$ years old $)$ originating from the German

315 Primate Center (Deutsches Primatenzentrum, Gottingen, Germany) were housed singly for the

316 duration of import and quarantine. At the original institution, during CDC import quarantine (31

317 d), and during quarantine at Johns Hopkins University, this population was fed a primary diet of

318 Mar V3843 (ssniff®, Soest, Germany).

320 populations remained quarantined from each other during CDC import quarantine, and were

321 housed in the same room but in original housing groups during the Johns Hopkins University

322 quarantine. The primary diet of populations DPG and HHG was 100\% Mar V3843 through

323 experimental day 1 (one day following arrival at JHU), 50\% V3843/50\% 5LK6 through

324 experimental day 50, and 100\% 5LK6 subsequently. The primary diet of the JHU population

325 remained unchanged $(100 \%$ 5LK6) throughout the study duration.

Adult animals in good clinical health were randomly assigned to this study from the three

327 populations (JHU, DPG, and HHG) following application of inclusion criteria: no history of

328 systemic or chronic disease, $2-8$ years of age, $\geq 3$ months since antimicrobial use (Table S1).

329 Age and body mass did not differ between groups at baseline (ANOVA; age: $\mathrm{F}=0.09, \mathrm{p}=0.91$;

330 body mass: $\mathrm{F}=0.06, \mathrm{p}=0.94)$.

331 Sampling procedure: JHU animals $(\mathrm{n}=18)$ were sampled once each during a two-month

332 window during Johns Hopkins University quarantine of populations DPG and HHG; no

333 introduction of new animals or changes in husbandry procedures took place during this duration. 
334 HHG animals $(n=15)$ and DPG animals $(n=18)$ were sampled less than 24 hours following

335 arrival at Johns Hopkins University (baseline). Sampling at the post-diet change time point (100

336 d) took place for populations 2 and 3; study participants from all three populations were sampled

337 at the $390 \mathrm{~d}$ time point, 8 months following full colony integration (Figure 1). microtube (Fisherbrand® Microcentrifuge Tubes, Fisher Scientific, Hampton, NH), flash-frozen

342 in liquid nitrogen, and stored at $-80^{\circ} \mathrm{C}$. All samples were collected between 7 am and 1 pm, prior 343 to feeding.

Out of 135 possible samples, 13 samples (9.6\%) were not included in analyses; reasons

345 included failure of subject to meet inclusion criteria at $100 \mathrm{~d}$ and/or $390 \mathrm{~d}(\mathrm{n}=5)$, death of

346 subject by $390 \mathrm{~d}(\mathrm{n}=3)$, transfer of study subject to an outside institution by $390 \mathrm{~d}(\mathrm{n}=1)$,

347 unavailability of sample $(n=2)$, or insufficient DNA yield of sample $(n=2)$.

DNA isolation and 16S rRNA gene sequencing analysis: DNA was extracted from thawed

349 samples using the QIAamp PowerFecal DNA Kit (QIAGEN, Hilden, Germany), and quantified

350 by the Qubit ${ }^{\circ}$ dsDNA HS Assay using a Qubit® 2.0 Fluorometer (Invitrogen, Life Technologies

351 Corporation, Carlsbad, CA). Illumina iTag Polymerase Chain Reactions (PCR) were performed

352 based on the Earth Microbiome Project's 16S rRNA amplification protocol. ${ }^{40}$ The volume of

353 each reaction was $25 \mu \mathrm{L}$ and contained (final concentrations) 1X PCR buffer, $0.8 \mathrm{mM}$ dNTP's,

$354 \quad 0.625 \mathrm{U}$ Ex Taq DNA Polymerase (Takara), $0.2 \mu \mathrm{M} 515 \mathrm{~F}$ barcoded forward primer, $0.2 \mu \mathrm{M}$

355 806R reverse primer and $\sim 10 \mathrm{ng}$ of template DNA per reaction. PCR was carried out on a T100

356 Thermal Cycler (Bio-Rad, Hercules, CA) using the following cycling conditions: $98{ }^{\circ} \mathrm{C}$ for 3 
min; then 35 cycles of $98^{\circ} \mathrm{C}$ for $1 \mathrm{~min}, 55^{\circ} \mathrm{C}$ for $40 \mathrm{~s}$, and $72{ }^{\circ} \mathrm{C}$ for $1 \mathrm{~min}$; final extension was at $72{ }^{\circ} \mathrm{C}$ for $10 \mathrm{~min}$; then held at $4{ }^{\circ} \mathrm{C}$. PCR products were visualized on a $2 \%$ agarose E-Gel with ethidium bromide (Thermo Fisher Scientific) for bands at $~ 400$ bp._Library pools were size verified using the Fragment Analyzer on the ABI3730 and were quantified with a KAPA Library quantification kit (Kapa Biosystem, Wilmington, MA, USA). After dilution with EBT (Illumina) to a final concentration of $2 \mathrm{nM}$ containing 15\% PhiX V3 library control (Illumina, San Diego, CA, USA), the library pools were denatured for $5 \mathrm{~min}$ in an equal volume of $0.2 \mathrm{M} \mathrm{NaOH}$, then further diluted to $8 \mathrm{pM}$ in HT1 buffer (Illumina) and were sequenced using an Illumina MiSeq V2 500 cycle kit cassette with $16 \mathrm{~S}$ rRNA library sequencing primers set for 250 base pair, paired-end reads. Overall sequencing run performance was evaluated by determining whether the sequencing run met the Illumina specifications for quality scores and data output. Actual run performance varied based on sample type, quality, and clusters passing filter. Specifications are based on the Illumina PhiX control library at supported cluster densities.

Bioinformatic Analysis: Paired-end sequences were imported into the DADA2 pipeline for

371 quality filtration, denoising, and chimera removal. ${ }^{41}$ Two hundred fifty base pair reads were

372 filtered at a maximum expected error of 1.0, with forward reads truncated at a length of 218, and

373 reverse at a length of 179. Trimmed sequences were then denoised, merged and underwent

374 chimera removal within DADA2. ${ }^{42}$ Taxonomy was then assigned against the SILVA database. ${ }^{43}$

375 ASVs were tabulated for use in downstream taxonomic summary and analysis. A reformatted

376 ASV-table was converted to BIOM format for use with QIIME. ${ }^{44}$

Alpha diversity box plots were generated within the QIIME2 sequence analysis package using an unrarified taxonomy table. ${ }^{45}$ Nonparametric t-test comparisons between experimental 
380 Sum Scaled (CSS) normalized counts of ASVs were imported into QIIME2 after which Principal

381 Coordinates Analysis (PCoA) plots were generated from calculated weighted UniFrac distance

382 matrices. Significance of clustering between samples within all PCoA plots was assessed using

383 the ANOSIM test statistic. For Linear Discriminant Analysis Effect Size (LEfSe) biomarker

384 analysis, relative abundances of bacterial taxa were multiplied by 1 million and formatted as

385 described in Segata et al. ${ }^{46}$ Alpha levels of 0.05 were used for both the Kruskal-Wallis and

386 pairwise Wilcoxon tests. Spearman's correlational analyses were conducted within QIIME-1.9.1,

387 in which species level taxa summaries were correlated against select continuous metadata

388 variables. ANOVA tests were performed using VassarStats (vassarstats.net) to determine

389 whether key characteristics (i.e. age, body mass) differed between experimental groups at study

390 initiation; there were no differences between groups $(\mathrm{p}>0.05)$. Functional predictions were

391 generated using the PICRUSt2 informatics package from generated ASVs. ${ }^{47}$ Predictions were

392 summarized at the pathway level (L3) and were subject to downstream LEfSe biomarker

393 analysis.

394 Data Availability: Sequencing data has been submitted to the NCBI Sequence Read Archive 395 with BioProject ID PRJNA659800. 


\section{Acknowledgments}

398 (GLAS) from the American Association for Laboratory Animal Science, NIH grant T32

399 OD011089, and Research Animal Resources at Johns Hopkins University School of Medicine.

400 The funders had no role in study design, data collection or interpretation, or the decision to

401 submit the work for publication. The authors thank Drs. Caroline Garrett and Jessica Izzi for

402 veterinary oversight and consultation throughout the project. We extend special thanks to Dr.

403 Xiaoqin Wang and his research team for generously sharing use of their animals, supported by

404 NIH grants U24MH123423, DC003180, DC005808, and DC014503 (PI: Wang). 


\section{References}

406

407

408

409

410

411

412

413

414

415

416

417

418

419

420

421

422

423

424

425

426

427

428

429

430

431

432

433

434

435

436

437

438

439

440

441

442

443

444

445

446

447

448

449

450

451

1. Servick K. Why are U.S. neuroscientists clamoring for marmosets? Science. October 23, 2018, 2018.

2. Malukiewicz J. CRA, Dergam J. A., Igayara C. S., Kessler S., Moreira S. B., Nash L. T., Nicola P. A., Pereira L. C. M., Pissinati A., Ruiz-Miranda C. R., Ozga A. T., Roos C., Silva D. L., Stone A. C., Grativol A. D. The Effects of Host Taxon, Hybridization, and Environment on the Gut Microbiome of Callithrix Marmosets. 2019.

3. Rasmussen TS, de Vries L, Kot W, Hansen LH, Castro-Mejia JL, Vogensen FK, Hansen AK, Nielsen DS. Mouse Vendor Influence on the Bacterial and Viral Gut Composition Exceeds the Effect of Diet. Viruses. 2019;11(5).

4. Hansen AK, Hansen CH, Krych L, Nielsen DS. Impact of the gut microbiota on rodent models of human disease. World J Gastroenterol. 2014;20(47):17727-17736.

5. Hilbert T, Steinhagen F, Senzig S, Cramer N, Bekeredjian-Ding I, Parcina M, Baumgarten G, Hoeft A, Frede $\mathrm{S}$, Boehm $\mathrm{O}$, Klaschik S. Vendor effects on murine gut microbiota influence experimental abdominal sepsis. J Surg Res. 2017;211:126-136.

6. Sadler R, Singh V, Benakis C, Garzetti D, Brea D, Stecher B, Anrather J, Liesz A. Microbiota differences between commercial breeders impacts the post-stroke immune response. Brain Behav Immun. 2017;66:23-30.

7. Moskowitz JE, Andreatta F, Amos-Landgraf J. The gut microbiota modulates differential adenoma suppression by $\mathrm{B} 6 / \mathrm{J}$ and $\mathrm{B} 6 / \mathrm{N}$ genetic backgrounds in $\mathrm{Apc}(\mathrm{Min})$ mice. Mamm Genome. 2019;30(9-10):237-244.

8. National Academies of Sciences E, Medicine. Care, Use, and Welfare of Marmosets as Animal Models for Gene Editing-Based Biomedical Research: Proceedings of a Workshop. Washington, DC: The National Academies Press; 2019.

9. Kap YS, Bus-Spoor C, van Driel N, Dubbelaar ML, Grit C, Kooistra SM, Fagrouch ZC, Verschoor EJ, Bauer J, Eggen BJL, Harmsen HJM, Laman JD, t Hart BA. Targeted Diet Modification Reduces Multiple Sclerosis-like Disease in Adult Marmoset Monkeys from an Outbred Colony. J Immunol. 2018;201(11):3229-3243.

10. Hsiao EY, McBride SW, Hsien S, Sharon G, Hyde ER, McCue T, Codelli JA, Chow J, Reisman SE, Petrosino JF, Patterson PH, Mazmanian SK. Microbiota modulate behavioral and physiological abnormalities associated with neurodevelopmental disorders. Cell. 2013;155(7):1451-1463.

11. Yano JM, Yu K, Donaldson GP, Shastri GG, Ann P, Ma L, Nagler CR, Ismagilov RF, Mazmanian SK, Hsiao EY. Indigenous bacteria from the gut microbiota regulate host serotonin biosynthesis. Cell. 2015;161(2):264-276.

12. Erny D, Hrabe de Angelis AL, Jaitin D, Wieghofer P, Staszewski O, David E, Keren-Shaul H, Mahlakoiv T, Jakobshagen K, Buch T, Schwierzeck V, Utermohlen O, Chun E, Garrett WS, McCoy KD, Diefenbach A, Staeheli P, Stecher B, Amit I, Prinz M. Host microbiota constantly control maturation and function of microglia in the CNS. Nat Neurosci. 2015;18(7):965-977.

13. Sampson TR, Mazmanian SK. Control of brain development, function, and behavior by the microbiome. Cell Host Microbe. 2015;17(5):565-576.

14. O'Mahony SM, Clarke G, Borre YE, Dinan TG, Cryan JF. Serotonin, tryptophan metabolism and the brain-gut-microbiome axis. Behav Brain Res. 2015;277:32-48.

15. Zheng P, Zeng B, Liu M, Chen J, Pan J, Han Y, Liu Y, Cheng K, Zhou C, Wang H, Zhou X, Gui S, Perry SW, Wong ML, Licinio J, Wei H, Xie P. The gut microbiome from patients with schizophrenia modulates the glutamate-glutamine-GABA cycle and schizophrenia-relevant behaviors in mice. Sci Adv. 2019;5(2):eaau8317. 
16. Zhu F, Guo R, Wang W, Ju Y, Wang $Q$, Ma Q, Sun $Q$, Fan $Y$, Xie $Y$, Yang Z, Jie Z, Zhao B, Xiao L, Yang L, Zhang T, Liu B, Guo L, He X, Chen Y, Chen C, Gao C, Xu X, Yang H, Wang J, Dang Y, Madsen L, Brix S, Kristiansen K, Jia H, MaX. Transplantation of microbiota from drug-free patients with schizophrenia causes schizophrenia-like abnormal behaviors and dysregulated kynurenine metabolism in mice. Mol Psychiatry. 2019.

17. Vogt NM, Kerby RL, Dill-McFarland KA, Harding SJ, Merluzzi AP, Johnson SC, Carlsson CM, Asthana S, Zetterberg H, Blennow K, Bendlin BB, Rey FE. Gut microbiome alterations in Alzheimer's disease. Sci Rep. 2017;7(1):13537.

18. Blacher E, Bashiardes S, Shapiro H, Rothschild D, Mor U, Dori-Bachash M, Kleimeyer C, Moresi C, Harnik Y, Zur M, Zabari M, Brik RB, Kviatcovsky D, Zmora N, Cohen Y, Bar N, Levi I, Amar N, Mehlman T, Brandis A, Biton I, Kuperman Y, Tsoory M, Alfahel L, Harmelin A, Schwartz M, Israelson A, Arike L, Johansson MEV, Hansson GC, Gotkine M, Segal E, Elinav E. Potential roles of gut microbiome and metabolites in modulating ALS in mice. Nature. 2019;572(7770):474-480.

19. Sharon G, Cruz NJ, Kang DW, Gandal MJ, Wang B, Kim YM, Zink EM, Casey CP, Taylor BC, Lane CJ, Bramer LM, Isern NG, Hoyt DW, Noecker C, Sweredoski MJ, Moradian A, Borenstein E, Jansson JK, Knight R, Metz TO, Lois C, Geschwind DH, Krajmalnik-Brown R, Mazmanian SK. Human Gut Microbiota from Autism Spectrum Disorder Promote Behavioral Symptoms in Mice. Cell. 2019;177(6):1600-1618 e1617.

20. Kishi N, Sato K, Sasaki E, Okano H. Common marmoset as a new model animal for neuroscience research and genome editing technology. Dev Growth Differ. 2014;56(1):53-62.

21. Tomioka I, Ishibashi H, Minakawa EN, Motohashi HH, Takayama O, Saito Y, Popiel HA, Puentes S, Owari K, Nakatani T, Nogami N, Yamamoto K, Noguchi S, Yonekawa T, Tanaka Y, Fujita N, Suzuki H, Kikuchi H, Aizawa S, Nagano S, Yamada D, Nishino I, Ichinohe N, Wada K, Kohsaka S, Nagai Y, Seki K. Transgenic Monkey Model of the Polyglutamine Diseases Recapitulating Progressive Neurological Symptoms. eNeuro. 2017;4(2).

22. Yasuda K, Oh K, Ren B, Tickle TL, Franzosa EA, Wachtman LM, Miller AD, Westmoreland SV, Mansfield KG, Vallender EJ, Miller GM, Rowlett JK, Gevers D, Huttenhower C, Morgan XC. Biogeography of the intestinal mucosal and lumenal microbiome in the rhesus macaque. Cell Host Microbe. 2015;17(3):385-391.

23. Montonye DR, Ericsson AC, Busi SB, Lutz C, Wardwell K, Franklin CL. Acclimation and Institutionalization of the Mouse Microbiota Following Transportation. Front Microbiol. 2018;9:1085.

24. Grieneisen LE, Charpentier MJE, Alberts SC, Blekhman R, Bradburd G, Tung J, Archie EA. Genes, geology and germs: gut microbiota across a primate hybrid zone are explained by site soil properties, not host species. Proc Biol Sci. 2019;286(1901):20190431.

25. Waite DW, Vanwonterghem I, Rinke C, Parks DH, Zhang Y, Takai K, Sievert SM, Simon J, Campbell BJ, Hanson TE, Woyke T, Klotz MG, Hugenholtz P. Erratum: Addendum: Comparative Genomic Analysis of the Class Epsilonproteobacteria and Proposed Reclassification to Epsilonbacteraeota (phyl. nov.). Front Microbiol. 2018;9:772.

26. Waite DW, Vanwonterghem I, Rinke C, Parks DH, Zhang Y, Takai K, Sievert SM, Simon J, Campbell BJ, Hanson TE, Woyke T, Klotz MG, Hugenholtz P. Comparative Genomic Analysis of the Class Epsilonproteobacteria and Proposed Reclassification to Epsilonbacteraeota (phyl. nov.). Front Microbiol. 2017;8:682.

27. Shen Z, Feng Y, Sheh A, Everitt J, Bertram F, Paster BJ, Fox JG. Isolation and characterization of a novel Helicobacter species, Helicobacter jaachi sp. nov., from common marmosets (Callithrix jaachus). J Med Microbiol. 2015;64(9):1063-1073. disease. Microbiome. 2017;5(1):80. 
29. Biagi E, Nylund L, Candela M, Ostan R, Bucci L, Pini E, Nikkila J, Monti D, Satokari R, Franceschi C, Brigidi $P$, De Vos W. Through ageing, and beyond: gut microbiota and inflammatory status in seniors and centenarians. PLoS One. 2010;5(5):e10667.

30. Jeffery IB, Lynch DB, O'Toole PW. Composition and temporal stability of the gut microbiota in older persons. ISME J. 2016;10(1):170-182.

31. Reveles KR, Patel S, Forney L, Ross CN. Age-related changes in the marmoset gut microbiome. Am J Primatol. 2019;81(2):e22960.

32. Ley RE, Turnbaugh PJ, Klein S, Gordon JI. Microbial ecology: human gut microbes associated with obesity. Nature. 2006;444(7122):1022-1023.

33. Kondo S, Kamei A, Xiao JZ, Iwatsuki K, Abe K. Bifidobacterium breve B-3 exerts metabolic syndrome-suppressing effects in the liver of diet-induced obese mice: a DNA microarray analysis. Benef Microbes. 2013;4(3):247-251.

34. Minami J, Iwabuchi N, Tanaka M, Yamauchi K, Xiao JZ, Abe F, Sakane N. Effects of Bifidobacterium breve B-3 on body fat reductions in pre-obese adults: a randomized, doubleblind, placebo-controlled trial. Biosci Microbiota Food Health. 2018;37(3):67-75.

35. Schwiertz A, Taras D, Schafer K, Beijer S, Bos NA, Donus C, Hardt PD. Microbiota and SCFA in lean and overweight healthy subjects. Obesity (Silver Spring). 2010;18(1):190-195.

36. Kalliomaki M, Collado MC, Salminen S, Isolauri E. Early differences in fecal microbiota composition in children may predict overweight. Am J Clin Nutr. 2008;87(3):534-538.

37. Kim YS, Unno T, Kim BY, Park MS. Sex Differences in Gut Microbiota. World J Mens Health. 2020;38(1):48-60.

38. Elderman M, Hugenholtz F, Belzer C, Boekschoten M, van Beek A, de Haan B, Savelkoul H, de Vos $\mathrm{P}$, Faas $\mathrm{M}$. Sex and strain dependent differences in mucosal immunology and microbiota composition in mice. Biol Sex Differ. 2018;9(1):26.

39. Kovacs A, Ben-Jacob N, Tayem H, Halperin E, Iraqi FA, Gophna U. Genotype is a stronger determinant than sex of the mouse gut microbiota. Microb Ecol. 2011;61(2):423-428.

40. Walters W, Hyde ER, Berg-Lyons D, Ackermann G, Humphrey G, Parada A, Gilbert JA, Jansson JK, Caporaso JG, Fuhrman JA, Apprill A, Knight R. Improved Bacterial 16S rRNA Gene (V4 and V4-5) and Fungal Internal Transcribed Spacer Marker Gene Primers for Microbial Community Surveys. mSystems. 2016;1(1).

41. Edgar RC. Search and clustering orders of magnitude faster than BLAST. Bioinformatics. 2010;26(19):2460-2461.

42. Callahan BJ, McMurdie PJ, Rosen MJ, Han AW, Johnson AJ, Holmes SP. DADA2: High-resolution sample inference from Illumina amplicon data. Nat Methods. 2016;13(7):581-583.

43. Quast C, Pruesse E, Yilmaz P, Gerken J, Schweer T, Yarza P, Peplies J, Glockner FO. The SILVA ribosomal RNA gene database project: improved data processing and web-based tools. Nucleic Acids Res. 2013;41(Database issue):D590-596.

44. Caporaso JG, Lauber CL, Walters WA, Berg-Lyons D, Lozupone CA, Turnbaugh PJ, Fierer N, Knight R. Global patterns of $16 \mathrm{~S}$ rRNA diversity at a depth of millions of sequences per sample. Proc Natl Acad Sci U S A. 2011;108 Suppl 1:4516-4522.

45. Bolyen E, Rideout JR, Dillon MR, et al. Reproducible, interactive, scalable and extensible microbiome data science using QIIME 2. Nat Biotechnol. 2019;37, 852-857.

46. Segata N, Izard J, Waldron L, Gevers D, Miropolsky L, Garrett WS, Huttenhower C. Metagenomic biomarker discovery and explanation. Genome Biol. 2011;12(6):R60.

47. Douglas G. M. MVJ, Saneveld J., Yurgel S. N., Brown J. R., Taylor C. M., Huttenhower C., Langille M. G. I. PICRUSt2: An improved and extensible approach for metagenome inference. bioRxiv. 2019. 


\section{$547 \quad$ Figures and Tables}

548

o d: Arrival
of DPG and
$H H G$ at

50 d: Diet

transition

JHU facility $\quad D P G$ and $H H G$
130-390 d: Integration of

$D P G$ and $H H G$ marmosets

with JHU colony
Baseline sample (DPG, HHG, JHU)

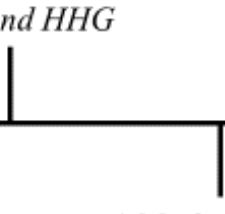

100 d sample

(DPG, HHG)
390 d sample

(DPG, HHG, JHU)

Figure 1. Experimental timeline. Two cohorts of common marmosets from captive research colonies in Germany (DPG, HHG) were imported to the United States and housed at an academic research institution with an existing captive colony (JHU). German-origin marmosets were transitioned from original diet (Mar V3843) onto JHU diet (Callitrichid Diet 5LK6) between days 1 and 50, and were released from quarantine and housed in three rooms containing the entirety of the JHU colony beginning on day 130 . Rectal swab samples were collected as indicated at 
A)

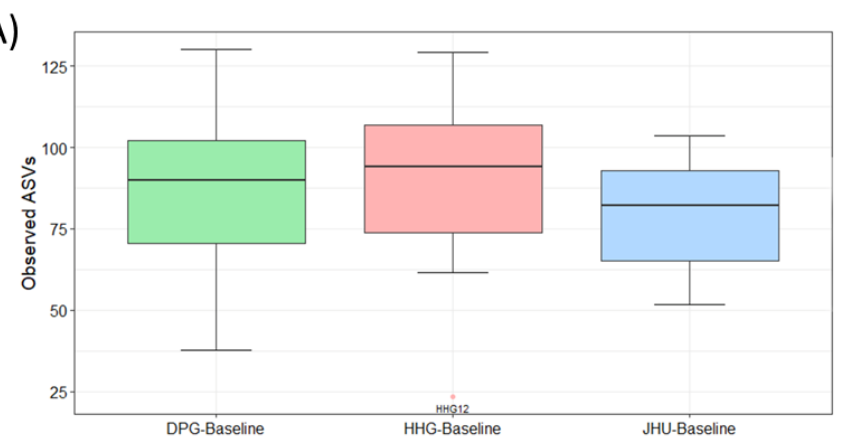

C)
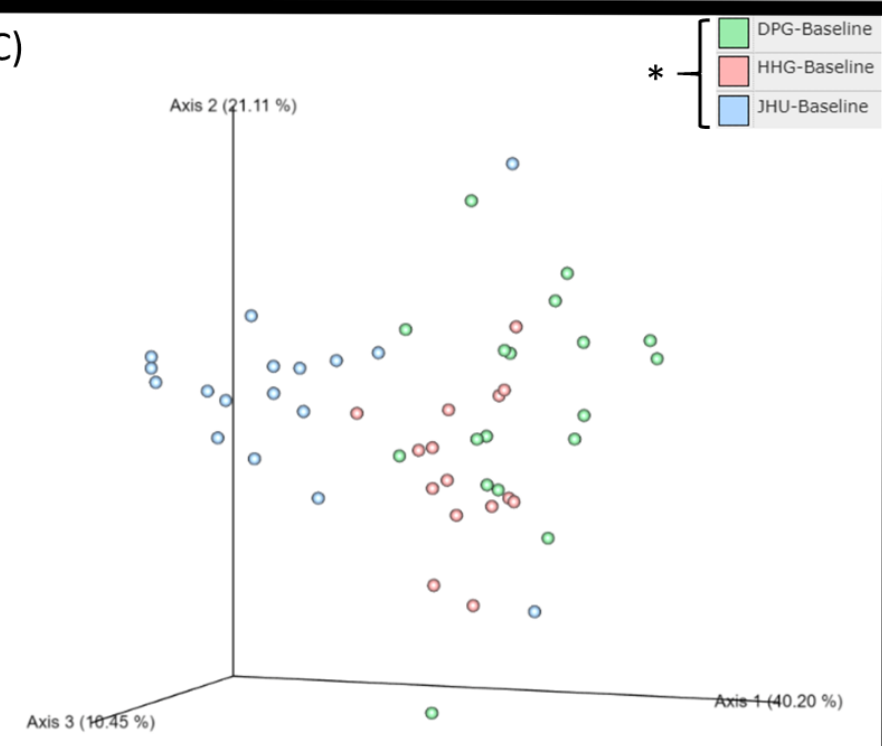

B)

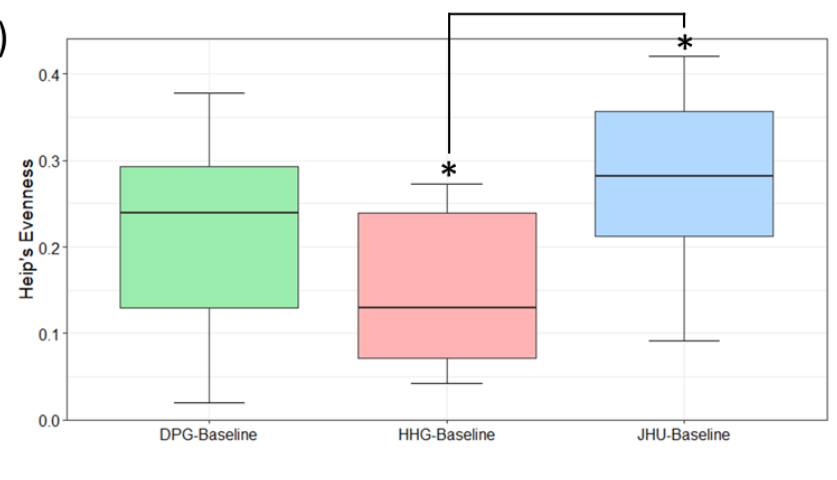

D)
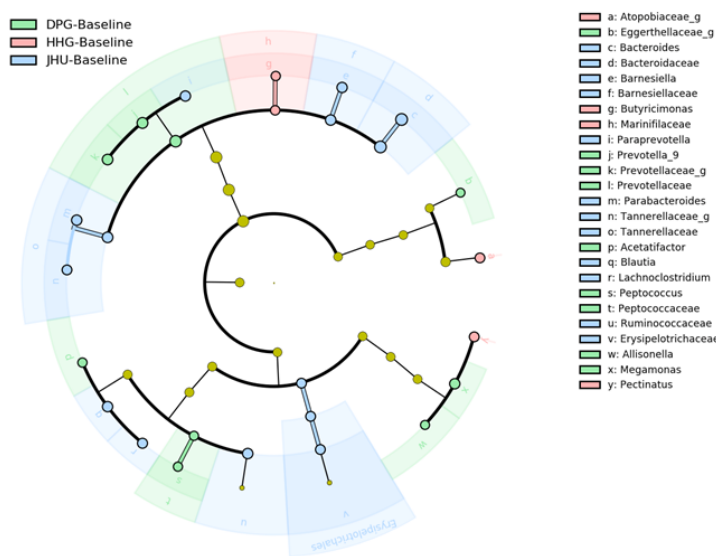

Figure 2. Effect of originating institution on the rectal bacterial community of captive common marmosets. A) Box plot of observed ASVs (alpha-diversity) in the DPG, HHG, and JHU populations at baseline demonstrates no difference between any cohorts. B) Box plot of Heip's Evenness (alpha-diversity) comparing DPG, HHG, and JHU populations at baseline demonstrates a significant difference between HHG and JHU $(p=0.006)$. C) PCoA plot with PERMANOVA demonstrates distinct rectal bacterial community structure (beta-diversity) between DPG, HHG, and JHU populations at baseline $(p=0.001)$. D) Cladogram depicts bacterial taxa that are more abundantly present in DPG $(n=9)$, HHG $(n=4)$, and JHU $(n=12)$ cohorts at baseline. (LDA > 2.5). $*=\mathrm{P}<0.05$. ASV $=$ amplicon sequence variants. $\mathrm{PCo} A=$ principle coordinates analysis. PERMANOVA = permutational analysis of variance. $L D A=$ linear discriminant analysis. 

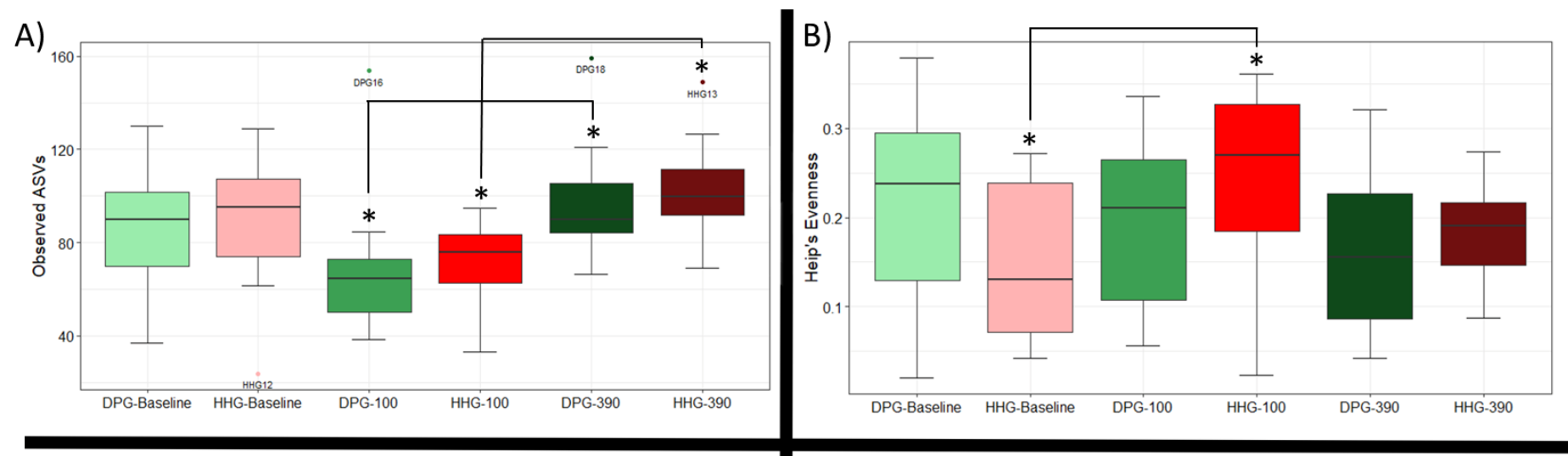

C)

D)
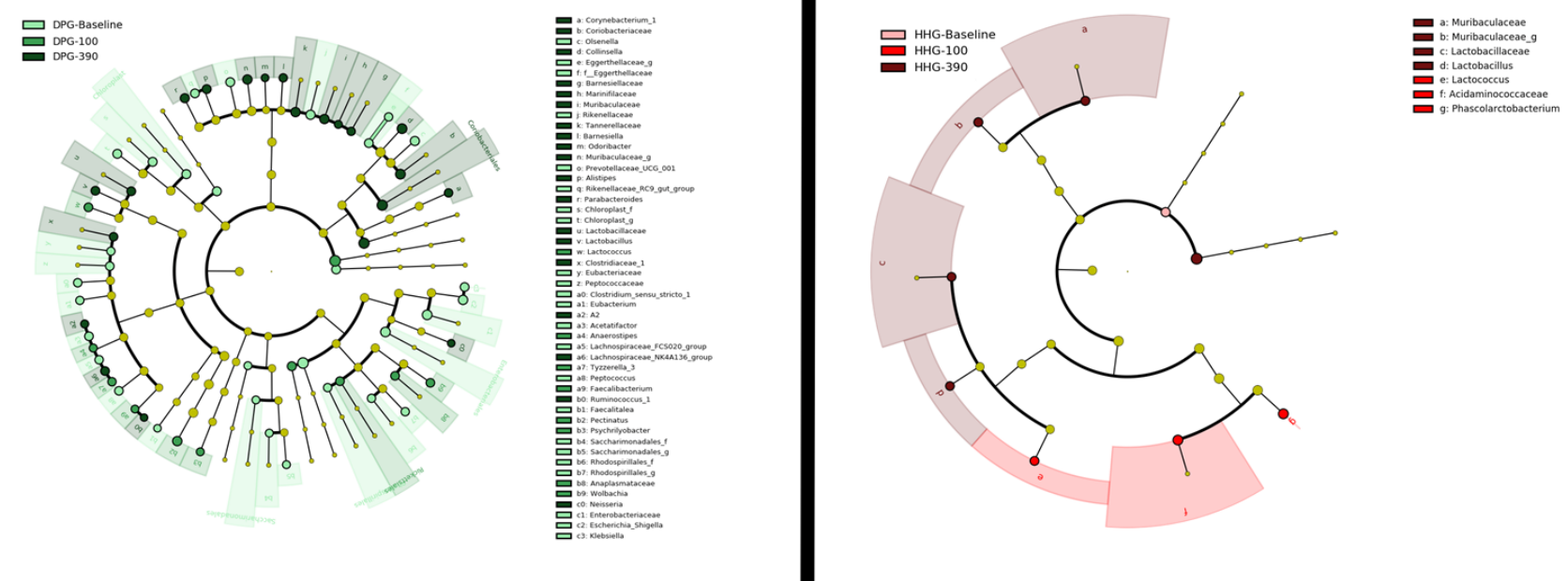

Figure 3. Changes in the rectal bacterial community in imported captive common marmosets (DPG and HHG) from baseline to $390 \mathrm{~d}$. A) Box plot of observed ASVs (alpha-diversity) in the DPG (green) and HHG (red) populations at baseline (lightest color saturation), $100 \mathrm{~d}$ (medium), and $390 \mathrm{~d}$ (dark). In the HHG and DPG cohorts, observed ASV richness is significantly increased between 100 days post-arrival and 390 d post-arrival (HHG $p=0.003$; DPG $p=0.01$ ). B) Box plot of Heip's evenness (alpha-diversity) in the bacterial community of DPG and HHG populations at baseline, $100 \mathrm{~d}$, and $390 \mathrm{~d}$. In the HHG cohort, there is increased Heip's evenness from baseline to the 100 day time point $(p=0.045)$. C) Cladogram depicts bacterial taxa that are more abundantly present at $100 d(n=3)$ and $390 d(n=4)$ in the DPG cohort (LDA > 2.5). D) Cladogram depicts bacterial taxa that are more abundantly present at baseline $(n=23), 100 d(n$ $=8)$, and $390 \mathrm{~d}(\mathrm{n}=19)$ in the HHG cohort (LDA > 2.5). ${ }^{*}=\mathrm{P}<0.05$. ASV = amplicon sequence variants. $\mathrm{LDA}=$ linear discriminant analysis. 
A)
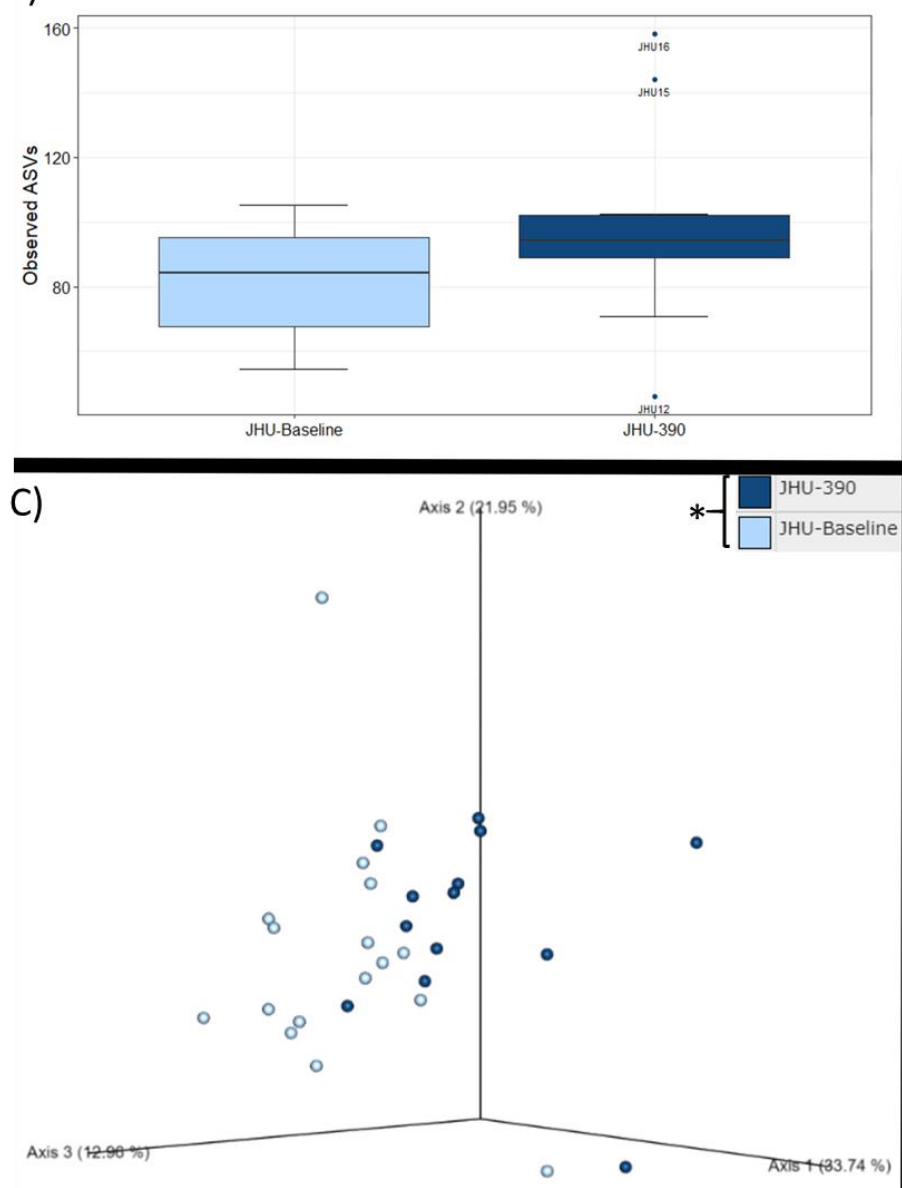

B)

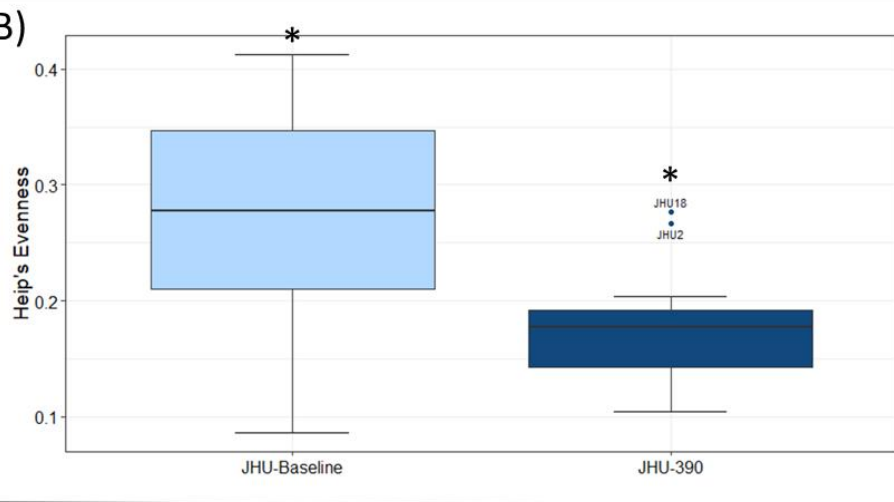

D)
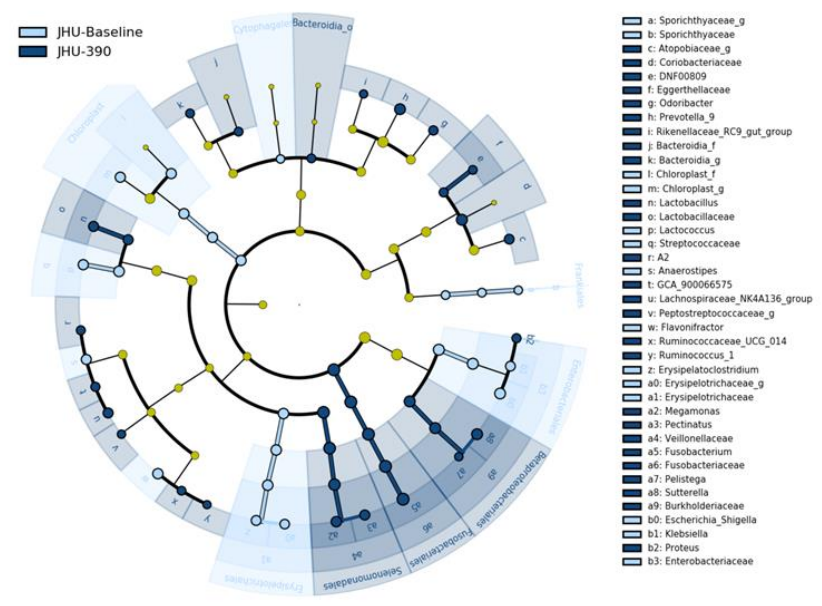

Figure 4. Changes in the rectal bacterial community in a stable population of captive common marmosets (JHU) from baseline to $390 \mathrm{~d}$. A) Box plot of observed ASVs (alpha-diversity) in the JHU cohort shows no change from baseline (light blue) to $390 \mathrm{~d}$ (dark blue) $(p=0.09)$. B) In the $\mathrm{JHU}$ cohort, bacterial evenness (Heip's evenness) is significantly decreased the $390 \mathrm{~d}$ time point compared to baseline $(p=0.002)$. C) PCoA plot with PERMANOVA demonstrates distinct bacterial community structure (beta-diversity) between baseline and $390 \mathrm{~d}$ in the JHU population $(p=0.022)$. D) Cladogram depicts bacterial taxa that are more abundantly present at baseline $(n=14)$ and $390 \mathrm{~d}(n=26)$ in the JHU cohort (LDA $>2.5) .{ }^{*}=p<0.05$. ASV = amplicon sequence variants. $\mathrm{PCOA}=$ principle coordinates analysis. $\mathrm{PERMANOVA}=$ permutational analysis 
A)

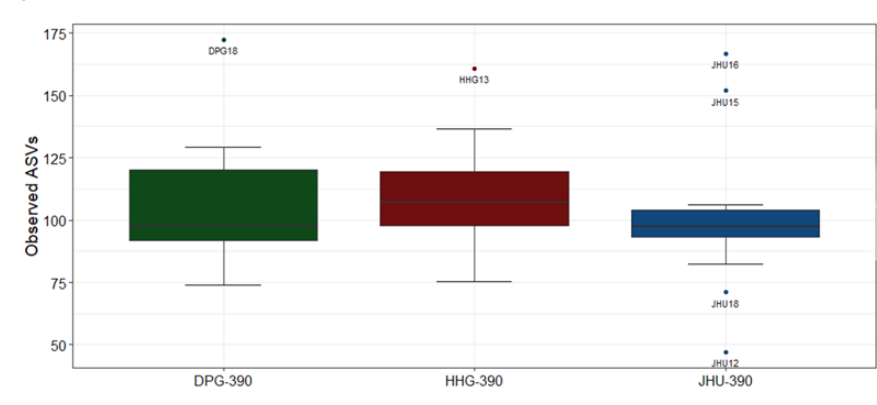

C)

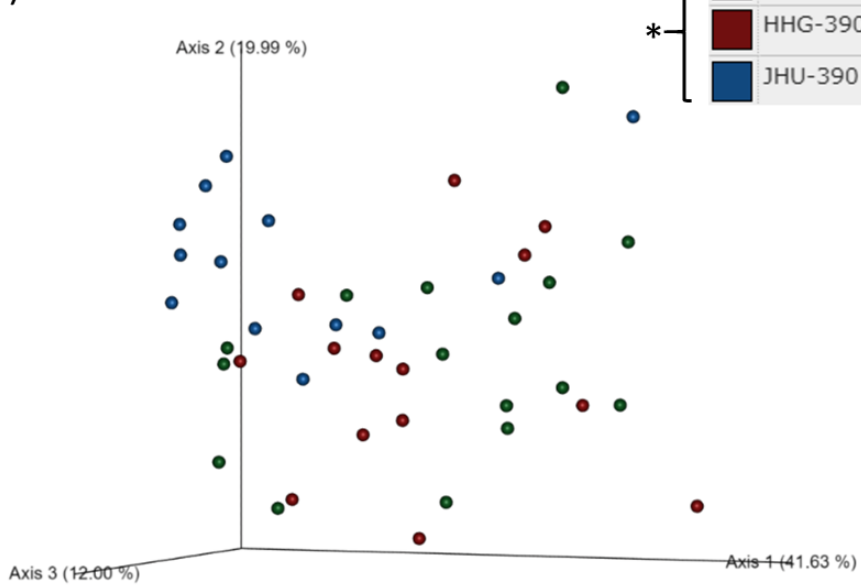

B)

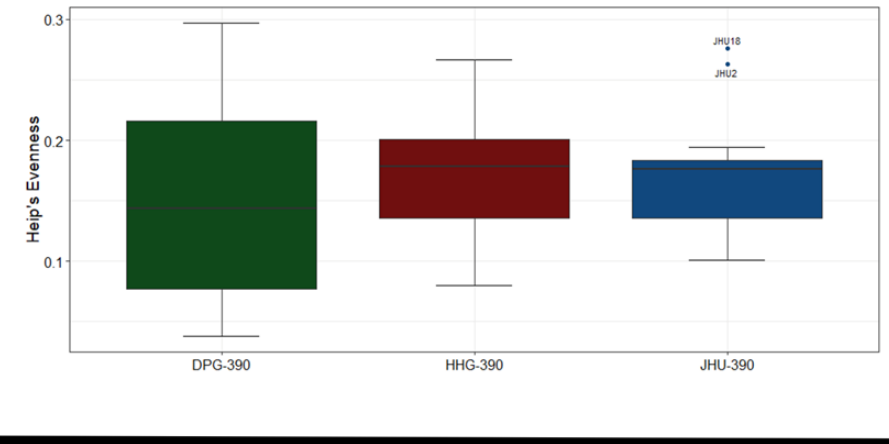

D)

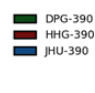

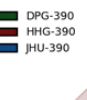
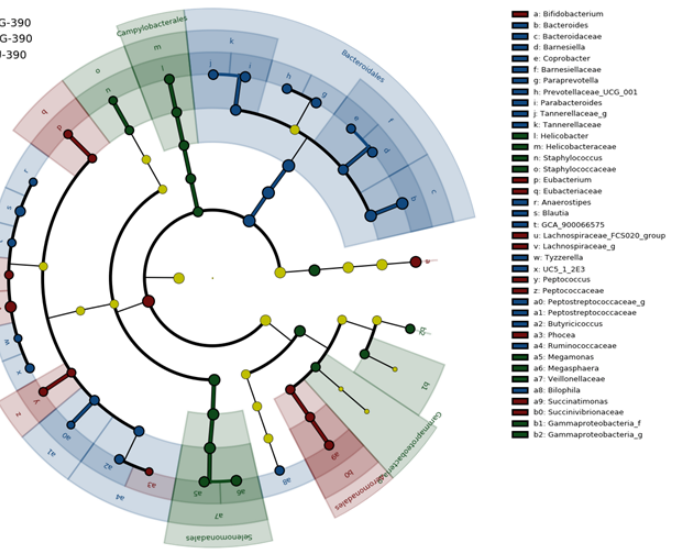

Figure 5. Effect of originating institution (DPG, HHG, JHU) on the rectal bacterial community of common marmosets at 390 d. A,B) Box plots of observed ASVS and Heip's Evenness (alphadiversity) in the HHG, DPG, and JHU common marmoset populations at $390 \mathrm{~d}$ demonstrate no difference between any cohorts. C) PCOA plot with PERMANOVA demonstrates distinct bacterial community structure (beta-diversity) between HHG, DPG, and JHU populations at $390 \mathrm{~d}$ ( $p=$ $0.002)$. D) Cladogram depicts bacterial taxa that are more abundantly present in DPG $(n=9)$, HHG $(n=10)$, and JHU $(n=20)$ cohorts at $390 d .{ }^{*}=p<0.05$. ASV = amplicon sequence variants.

608 $\mathrm{PCOA}=$ principle coordinate
linear discriminant analysis. 
A)

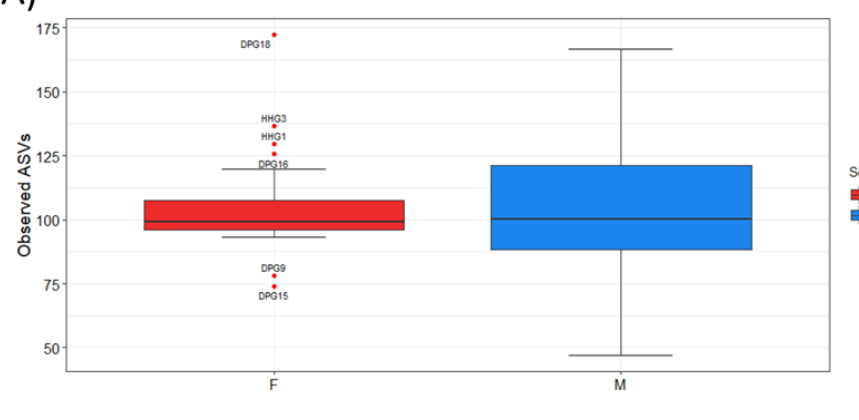

B)

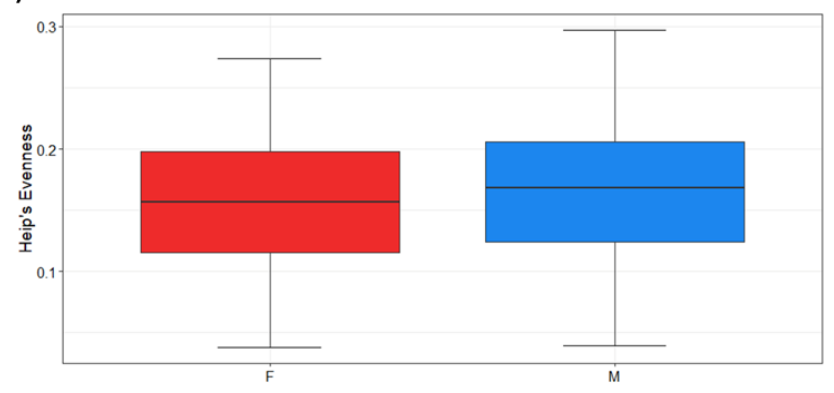

D)

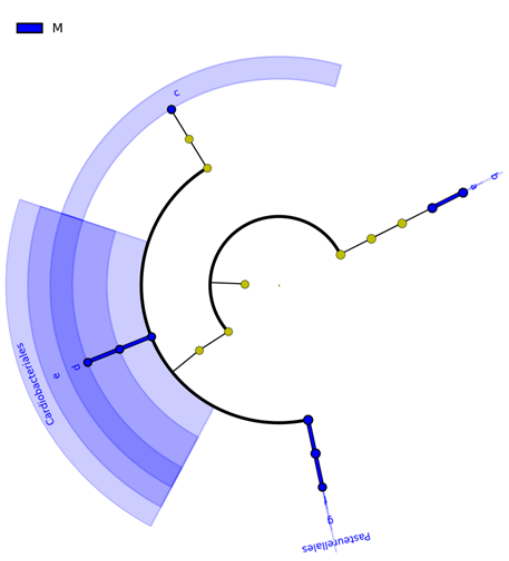

C)

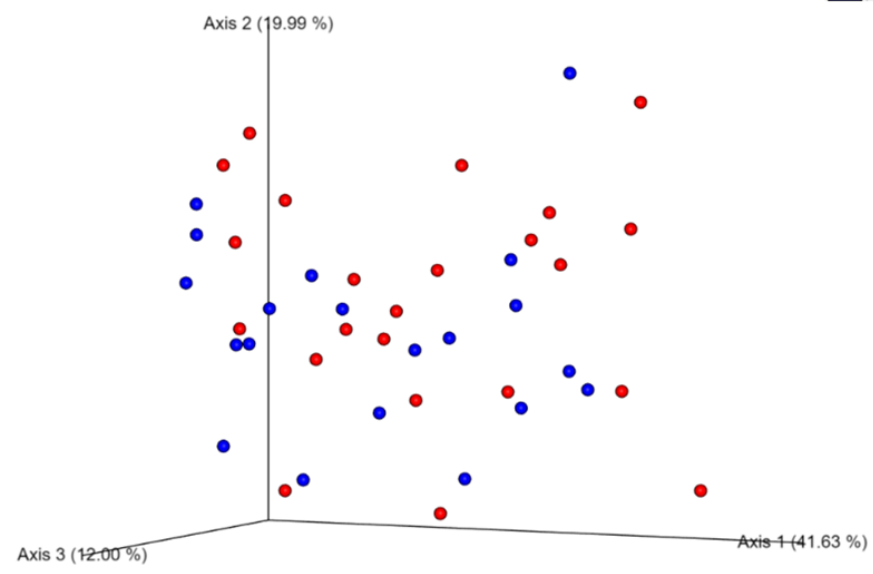

Figure 6. Effect of sex (male vs. female) on the rectal bacterial community of common marmosets at 390 d. Data from DPG, HHG, and JHU cohorts are grouped for analysis. A,B) Box plots of observed ASVS and Heip's Evenness (alpha-diversity) in male and female marmosets demonstrate no difference between sexes. C) PCoA plot with PERMANOVA demonstrates no difference in bacterial community composition between male and female marmosets $(p=$ $0.623)$. D) Cladogram depicts bacterial taxa that are more abundantly present in male marmosets $(n=7)(L D A>2.5)$. ASV $=$ amplicon sequence variants. $P C o A=$ principle coordinates analysis. PERMANOVA = permutational analysis of variance. $L D A=$ linear discriminant analysis. 


\begin{tabular}{l} 
Bacterial Phylum \\
\hline Actinobacteria \\
\hline Bacteroidetes \\
\hline Firmicutes \\
\hline Fusobacteria \\
\hline Proteobacteria (traditional classification) \\
\hline Proteobacteria (excludes Epsilonproteobacteria / Campylobacterota) \\
\hline Epsilonproteobacteria / Campylobacterota
\end{tabular}

\begin{tabular}{|c|c|}
\hline \multicolumn{2}{|c|}{ DPG (\%) } \\
\hline $\begin{array}{l}\text { Baseline } \\
(n=18)\end{array}$ & $\begin{array}{c}390 d \\
(n=16)\end{array}$ \\
\hline 0 & 0 \\
\hline 66.7 & 56.3 \\
\hline 0 & 6.3 \\
\hline 0 & 0 \\
\hline
\end{tabular}

Table 1. At the baseline and $390 \mathrm{~d}$ time points, percentage of common marmosets in the DPG, HHG, and JHU cohorts with rectal bacterial communities dominated by noted bacterial phyla. Campylobacterota is a proposed phylum comprising members of the class Epsilonproteobacteria 
bioRxiv preprint doi: https://doi.org/10.1101/2020.08.31.276733; this version posted September 1, 2020. The copyright holder for this preprint (which was not certified by peer review) is the author/funder, who has granted bioRxiv a license to display the preprint in perpetuity. It is made available under aCC-BY-NC-ND 4.0 International license.

\section{Supplemental Tables}

\begin{tabular}{|c|c|c|c|c|c|c|c|c|}
\hline Experimental Group & Animal ID & Sex & Age $(y)$ at baseline & Body mass (g) at baseline & $\begin{array}{l}\text { Housing status ( } \mathrm{S}=\text { single; } \mathrm{G}= \\
\text { group) }\end{array}$ & Baseline sample & $100 \mathrm{~d}$ sample & $390 \mathrm{~d}$ sample \\
\hline \multirow[t]{19}{*}{ DPG } & & & Mean: 4.36 & Mean: 405 & & $n=18$ & $n=15$ & $n=16$ \\
\hline & DPG15 & $\mathrm{F}$ & 3.55 & 415 & $\mathrm{~s}$ & yes & yes & yes \\
\hline & DPG7 & $\mathrm{F}$ & 6.2 & 420 & s & yes & yes & yes \\
\hline & DPG9 & $\mathrm{F}$ & 3.48 & 415 & s & yes & yes & yes \\
\hline & DPG16 & $\mathrm{F}$ & 3.5 & 415 & s & yes & yes & yes \\
\hline & DPG17 & $\mathrm{F}$ & 3.61 & 400 & $\mathrm{~s}$ & yes & yes & yes \\
\hline & DPG8 & $\mathrm{F}$ & 6.42 & 430 & s & yes & no & no \\
\hline & DPG2 & $\mathrm{F}$ & 3.33 & 370 & s & yes & yes & yes \\
\hline & DPG6 & $\mathrm{F}$ & 3.18 & 430 & s & yes & yes & yes \\
\hline & DPG18 & $\mathrm{F}$ & 3.5 & 465 & s & yes & yes & yes \\
\hline & DPG13 & M & 7.35 & 410 & $\mathrm{~s}$ & yes & yes & yes \\
\hline & DPG3 & M & 7.13 & 335 & $\mathrm{~s}$ & yes & no & no \\
\hline & DPG4 & M & 3.02 & 350 & s & yes & yes & yes \\
\hline & DPG11 & M & 4.47 & 435 & s & yes & yes & yes \\
\hline & DPG1 & M & 5.3 & 400 & $\mathrm{~s}$ & yes & yes & yes \\
\hline & DPG5 & M & 5.34 & 375 & s & yes & yes & yes \\
\hline & DPG10 & M & 2.81 & 385 & s & yes & yes & yes \\
\hline & DPG12 & M & 2.87 & 425 & $\mathrm{~s}$ & yes & no & yes \\
\hline & DPG14 & M & 3.48 & 415 & s & yes & yes & yes \\
\hline \multirow[t]{16}{*}{ HHG } & & & Mean: 4.15 & Mean: 401.33 & & $n=15$ & $n=14$ & $n=14$ \\
\hline & HHG10 & $\mathrm{F}$ & 3.82 & 420 & G & yes & yes & yes \\
\hline & HHG7 & $\mathrm{F}$ & 5.52 & 345 & $\mathrm{~s}$ & yes & no & no \\
\hline & HHG11 & $\mathrm{F}$ & 2.96 & 405 & G & yes & yes & yes \\
\hline & HHG1 & $\mathrm{F}$ & 5.52 & 370 & G & yes & yes & yes \\
\hline & HHG2 & $\mathrm{F}$ & 6.36 & 445 & G & yes & yes & yes \\
\hline & HHG8 & $\mathrm{F}$ & 6.79 & 420 & s & yes & yes & yes \\
\hline & HHG3 & $\mathrm{F}$ & 5.09 & 445 & G & yes & yes & yes \\
\hline & HHG9 & $\mathrm{F}$ & 4.67 & 405 & s & yes & yes & yes \\
\hline & HHG12 & $\mathrm{F}$ & 3.82 & 430 & G & yes & yes & yes \\
\hline & HHG4 & M & 4.24 & 375 & G & yes & yes & yes \\
\hline & HHG5 & M & 2.1 & 390 & G & yes & yes & yes \\
\hline & HHG13 & M & 2.96 & 380 & $\mathrm{~s}$ & yes & yes & yes \\
\hline & HHG6 & M & 3.39 & 425 & G & yes & yes & yes \\
\hline & HHG15 & M & 2.53 & 400 & s & yes & yes & yes \\
\hline & HHG14 & M & 2.53 & 365 & s & yes & yes & yes \\
\hline \multirow[t]{19}{*}{ JHU } & & & Mean: 4.60 & Mean: 382.44 & & $n=17$ & & $n=13$ \\
\hline & JHU5 & $\mathrm{F}$ & 2.92 & 372 & s & no & & Yes \\
\hline & JHU17 & $\mathrm{F}$ & 2.4 & 336 & s & Yes & & Yes \\
\hline & JHUG & $\mathrm{F}$ & 4.99 & 304 & s & Yes & & Yes \\
\hline & JHU13 & $\mathrm{F}$ & 6.75 & 366 & G & Yes & & no \\
\hline & JHU7 & $\mathrm{F}$ & 4.55 & 346 & G & Yes & & Yes \\
\hline & JHU4 & $\mathrm{F}$ & 5.03 & 456 & G & Yes & & Yes \\
\hline & JHU14 & $\mathrm{F}$ & 2.28 & 334 & $\mathrm{~s}$ & Yes & & Yes \\
\hline & JHU1 & $\mathrm{F}$ & 7.21 & 468 & G & Yes & & Yes \\
\hline & JHU9 & $\mathrm{F}$ & 2.54 & 364 & s & Yes & & no \\
\hline & JHU11 & M & 6.55 & 388 & s & Yes & & Yes \\
\hline & JHU16 & M & 6.33 & 330 & s & Yes & & Yes \\
\hline & JHU18 & M & 4.61 & 346 & G & Yes & & Yes \\
\hline & JHU3 & M & 2.19 & 490 & s & Yes & & no \\
\hline & JHU10 & M & 7.82 & 356 & s & Yes & & no \\
\hline & JHU12 & M & 6.38 & 386 & $\mathrm{~s}$ & Yes & & Yes \\
\hline & JHU2 & M & 2.25 & 410 & G & Yes & & Yes \\
\hline & JHU8 & M & 2.67 & 350 & G & Yes & & no \\
\hline & JHU15 & M & 5.28 & 482 & s & yes & & Yes \\
\hline
\end{tabular}




\begin{tabular}{|l|l|l|l|}
\hline Experimental Group & Baseline vs. 390 d & Baseline vs. 100 d & $100 \mathrm{~d}$ vs. $390 \mathrm{~d}$ \\
\hline DPG & $0.002^{*}$ & $0.007^{*}$ & 0.057 \\
\hline HHG & $0.020^{*}$ & $0.002^{*}$ & $0.021^{*}$ \\
\hline JHU & $0.011^{*}$ & & \\
\hline
\end{tabular}

Supplemental Table 2. Within-cohort comparisons of sample diversity (beta diversity) over time. FDR corrected $p$ values.

\begin{tabular}{|l|l|l|l|}
\hline $\begin{array}{l}\text { Experimental Time } \\
\text { Point }\end{array}$ & DPG vs. HHG & DPG vs. JHU & HHG vs. JHU \\
\hline Baseline & $0.005^{*}$ & $0.002^{*}$ & $0.002^{*}$ \\
\hline $100 \mathrm{~d}$ & 0.082 & $0.006^{*}$ & $0.004^{*}$ \\
\hline $390 \mathrm{~d}$ & 0.573 & & \\
\hline
\end{tabular}

Supplemental Table 4. Correlational analysis of rectal bacterial community characteristics associated with marmoset age at $390 \mathrm{~d}$. Spearman correlation detected age-associated alterations in two low-level taxa within the study population (HHG, DPG, and JHU) at $390 \mathrm{~d}$. A positive test statistic indicates positive correlation with increasing age.

k_Bacteria;p_Actinobacteria;c_Actinobacteria;o_Bifidobacteriales;f_Bifidobacteriaceae;g_NA;S_NA

k_Bacteria;p_Fusobacteria;c_Fusobacteriia;o_Fusobacteriales;f_Fusobacteriaceae;g_Fusobacterium;s_mortiferum

k_Bacteria;p_Firmicutes;c_Clostridia;o_Clostridiales;f_Ruminococcaceae;g__Butyricicoccus;s_NA

k_Bacteria;p_Actinobacteria;c_Actinobacteria;o_Bifidobacteriales;f__Bifidobacteriaceae;g_Bifidobacterium;s_aesculapii

k_Bacteria;P_Bacteroidetes;c_Bacteroidia;o__Bacteroidales;f_Muribaculaceae;g_NA;s_NA

k_Bacteria; $p \_$Actinobacteria;c_Coriobacteriia;o_Coriobacteriales;f__Atopobiaceae;g_NA;s_NA

k_Bacteria;p_Firmicutes;c_Clostridia;o_Clostridiales;f_Peptostreptococcaceae;g_NA;s_NA

\section{Supplemental Table 5. Correlational analysis of rectal bacterial community characteristics} associated with marmoset body mass at $390 \mathrm{~d}$. Spearman correlation detected body massassociated alterations in 13 low-level taxa within the study population (HHG, DPG, and JHU) at $390 \mathrm{~d}$. A positive test statistic indicates positive correlation with increasing body mass, and a negative test statistic indicates negative correlation. 